INPLASY

PROTOCOL

To cite: Fan et al. The effect of repetitive transcranial magnetic stimulation on lowerlimbmotor ability in stroke patients: A systematic review. Inplasy protocol 202180079. doi:

10.37766/inplasy2021.8.0079

Received: 21 August 2021

Published: 21 August 2021

Corresponding author:

Yaodong Gu

guyaodong@nbu.edu.cn

Author Affiliation:

Ningbo University

Support: Ningbo University.

Review Stage at time of this submission: Completed but not published.

Conflicts of interest:

None declared.

\section{The effect of repetitive transcranial magnetic stimulation on lower- limbmotor ability in stroke patients: A systematic review}

\author{
Fan, $\mathrm{H}^{1}$; Song, Y2; Cen, X3; Yu, P4; Bíró, 15; Gu, Y6.
}

Review question / Objective: This study aimed to evaluate the value of repetitive transcranial magnetic stimulation (rTMS) in the treatment of lower-limb motor dysfunction in stroke patients via gait characteristics.

Condition being studied: Repetitive transcranial magnetic stimulation (rTMS) is fundamental in inducing neuroplastic changes and promoting brain function restoration. Nevertheless, there is quite limited evidence based on the systematic assessment of the implication of rTMS in stroke patients up to now, let alone any conclusions regarding the better setting of rTMS interventions (e.g. frequency and duration) that could contribute to more benefit.

INPLASY registration number: This protocol was registered with the International Platform of Registered Systematic Review and Meta-Analysis Protocols (INPLASY) on 21 August 2021 and was last updated on 21 August 2021 (registration number INPLASY202180079).

\section{INTRODUCTION}

Review question / Objective: This study aimed to evaluate the value of repetitive transcranial magnetic stimulation (rTMS) in the treatment of lower-limb motor dysfunction in stroke patients via gait characteristics.
Rationale: Stroke is an acute syndrome of clinical signs of focal (or global) disturbance of cerebral function, which can even lead to death. It was indicated that gait performance is an important index to evaluate lower extremity motor function recovery poststroke. Repetitive transcranial magnetic stimulation (rTMS), one of the brain stimulation techniques 
without any trauma, can be used to induce neuroplastic changes as well as promote brain function restoration.

Condition being studied: Repetitive transcranial magnetic stimulation (rTMS) is fundamental in inducing neuroplastic changes and promoting brain function restoration. Nevertheless, there is quite limited evidence based on the systematic assessment of the implication of rTMS in stroke patients up to now, let alone any conclusions regarding the better setting of rTMS interventions (e.g. frequency and duration) that could contribute to more benefit.

\section{METHODS}

Search strategy: The search strategy was applied to find out all the relevant published literature on the influence of rTMS on lower limb motor dysfunction in stroke patients through ScienceDirect, Google Scholar, and PubMed using "repetitive transcranial magnetic stimulation" AND "gait" AND "stroke".

Participant or population: Participants who have been diagnosed with stroke were included in this study, and there is no restriction on age, sex, and ethnicity.

Intervention: Studies where participants received rTMS interventions or rTMS interventions combined with traditional physiotherapy and occupational therapy were included.

Comparator: There is no limitation regarding the control interventions.

Study designs to be included: English papers focus on the change of gait characteristics before and after interventions.

Eligibility criteria: English papers that published on peer-reviewed journals were covered, however, conference abstracts, review articles, book chapters, case reports, etc., were not included in this review.
Information sources: All included research on the influence of rTMS on lower limb motor dysfunction in stroke patients were searched through ScienceDirect, Google Scholar, and PubMed, and the citation snowballing method was applied to identify other potentially relevant studies in the reference list of all eligible articles. And these studies that have been accidentally overlooked were searched in other electronic databases to get available fulltext by entering the specific information of authors and article titles.

Main outcome(s): The main outcomes that collected from these included studies was the change of gait characteristics after interventions.

Data management: In order to ensure a rigorous searching process, two researchers independently searched and assessed the retrieved literature. Any disagreements of inclusion (if existed) would be resolved with the third author. Mendeley software (Elsevier Ltd., Amsterdam, Netherlands) was applied to organize published literature and create citations. Any disagreements were handled by discussing or consulting with another author.

Quality assessment / Risk of bias analysis: Two researchers independently evaluated the quality of these included studies. More specifically, seven following aspects were assessed, 1) random sequence generation; 2) allocation concealment; 3) blinding of participants and personnel; 4)blinding of outcome assessment; 5) incomplete outcome data; 6) selective reporting; 7) other biases. Any disagreements were handled by discussing or consulting with another author.

Strategy of data synthesis: For each published literature, the following information was extracted and summarized, and verified by two authors independently, characteristics of studies (i.e. authors and year), characteristics of subjects (i.e. sample size, age, gender, time post stroke, and treatment state), experimental design (i.e. coil type, 
intervention, control, and parameters), and primary outcomes.

Subgroup analysis: The main findings of these included studies were further presented in the following two sections, 1) Effects of low-frequency stimulus; 2) Effects of high-frequency stimulus.

Sensitivity analysis: No sensitivity analysis.

Language: Only English papers were considered in this study.

Country(ies) involved: China.

Keywords: repetitive transcranial magnetic stimulation, stroke, walking, balance, systematic review.

Contributions of each author:

Author 1 - Huiliu Fan.

Author 2 - Yang Song.

Author 3 - Xuanzhen Cen.

Author 4 - Peimin Yu.

Author 5 - István Bíró.

Author 6 - Yaodong Gu. 